\title{
Prolonged Neuromuscular Blockade following a bolus of Succinylcholine and Rett syndrome: a Case Report
}

Spencer, L.; Perry da Câmara; L., Salta, C.; Poeira, R.; Casteleira, M.

INTRODUCTION

Rett syndrome is a rare, genetic, neurodevelopment disorder that affects mainly women ${ }^{1}$. It is caused by mutations in the gene that encodes methyl CpG binding protein 2 (MeCP2). This gene is located on the long arm ( $q$ ) of the $X$ chromossome, regulating the expression of several genes involved in neuronal physiology. ${ }^{2}$

This syndrome is characterized by the progressive loss of cognitive, motor and comunication capacities; seizures and EEG changes. It may also be associated with electrolyte imbalance, cardiopulmonary and skeletal muscle abnormalities and airway deformities. These features lead this disease into a challenging anesthetic management. ${ }^{3}$

\section{CASE REPORT}

- 38 year old woman

- Surgery: dental extractions (tooth decays)

- Rett Syndrome (diagnosed in her childhood)

- No past history of anesthesia or surgery

Preoperative Evaluation

- Rett Syndrome - stage 4: severe cognitive impairment, craniofacial dysmorphia, dorso lumbar scoliosis, hypotonia and limb spasticity

- Airway: limited evaluation due to patient condition

- Pulmonary Auscultation: normal

- Complementary Exams: normal

- Preoperative medication: midazolam per os $(0,15 \mathrm{mg} / \mathrm{kg})$

INTRA AND POSTOPERATIVE

- Induction: Rapid Sequence Induction: propofol ( $2 \mathrm{mg} / \mathrm{kg})$, fentanyl $(1 \mathrm{mcg} / \mathrm{kg})$ e succinylcholine $(1 \mathrm{mg} / \mathrm{kg})$

- Maintenance: sevoflurane

- Standard Monitoring (ASA)+ NMB monitoring by acceleromyography

- No hemodynamic instability during the surgery

- $\mathrm{TOF}=0$ one hour after the induction

- Complete reversal of the NMB (TOF>95\%) 90 minutes after succinylcholine administration

- Postoperative recovery without complications

- Further investigation was proposed by the Genetics Department refused by the patient's family

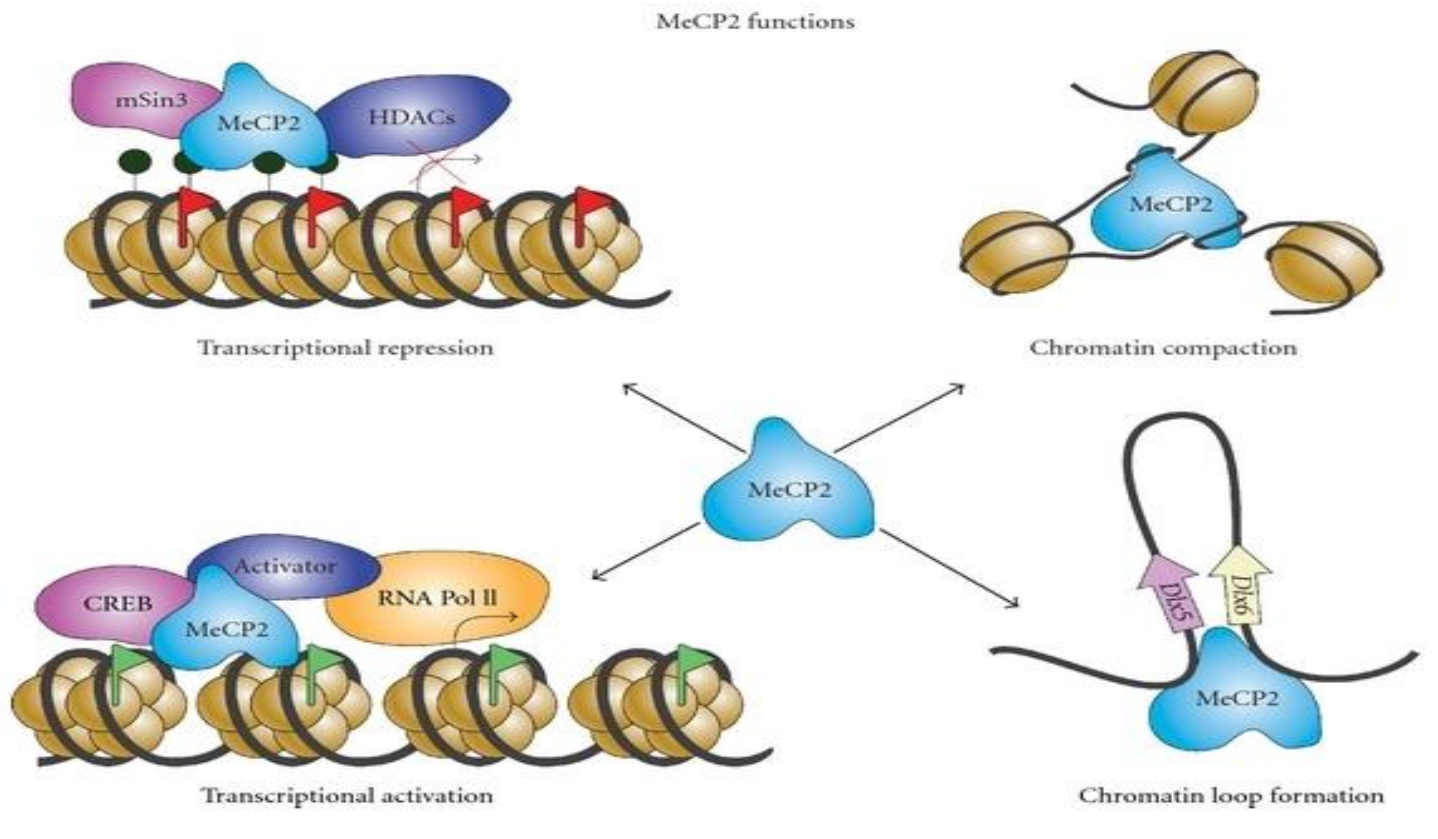

Image 1-MECP 2 functions

\section{Discussion}

- Rett Syndrome is a rare disease, that is why the optimal anesthetic management is not well defined. The choice of balanced general anesthesia for teeth extraction was due to the patient cognitive impairment. ${ }^{4}$

- Rett is associated with hyperkaliemia with the underlying risk of arrhythmia. The use of succinylcholine in Rett patients, though controversial, is not contraindicated.

- In this case, in the absence of hyperkaliemia in the preoperative laboratory values, succinylcholine was the neuromuscular drug chosen, provoking a prolonged neuromuscular block.

- Literature suggests the hypothesis of MeCP2 regulating, via microRNA (hsa-mIR-212 e 132), the expression of $\mathrm{BCHE}$ and $\mathrm{ACHE}$, that encode butyrylcholinesterase and acetylcholinesterase. A mutated MeCP2 would, therefore decrease butyrylcholinesterase and acetylcholinesterase transcription. ${ }^{5}$

- Non genetic factors causing prolonged NMB were excluded.

\section{CONCLUSION}

In patients with Rett Syndrome succinylcholine should be carefully used due to the risk of arrhythmia. This case suggest the risk that the prolonged NMB is also present, may be due to an enzimatic deficit caused by a genetic mutation. Further investigation is necessary to test this hypothesis. 